\title{
The influence of octenidine on human skin cells and wound healing
}

\author{
$\underline{\text { N. Nikolićć }}^{1}$, P. Kienzl ${ }^{1}$, P. Tajpara ${ }^{1}$, M. Mildner², J. Matiasek ${ }^{3}$, A. Elbe-Bürger ${ }^{1}$
}

${ }^{1}$ Department of Dermatology, Division of Immunology, Allergy and Infectious Diseases, Medical University of Vienna, Vienna, Austria

${ }^{2}$ Department of Dermatology, Research Division of Biology and Pathobiology of the Skin, Medical University of Vienna, Vienna, Austria

${ }^{3}$ Department of Plastic, Aesthetic and Reconstructive Surgery, St. Josef Hospital, Vienna, Austria

\section{Introduction \& Study Aim}

Prevention of infections by using antiseptics is a key element in professional wound management. Ideal agents for the topical treatment of skin wounds should have antimicrobial efficacy without negative influence on wound healing. Compared to other antiseptics (e.g. polihexanide, chlorhexidine, PVPlodine), octenidine dihydrochloride (OCT) shows high antimicrobial efficacy already within a short contact time even at low concentrations and remains active locally for more than 48 hours ("remanence effect").

To date, OCT has become a widely used antiseptic in modern wound care, but little is known about its effects on skin physiology. Results from animal studies and recent clinica observations lead to the hypothesis that besides its high antimicrobial effects, OCT could further have a positive influence on wound healing. Thus, we have tested its impact on immune and non-immune skin cells upon topical application on ex vivo normal and wounded human skin explants.

\section{Materials \& Methods}
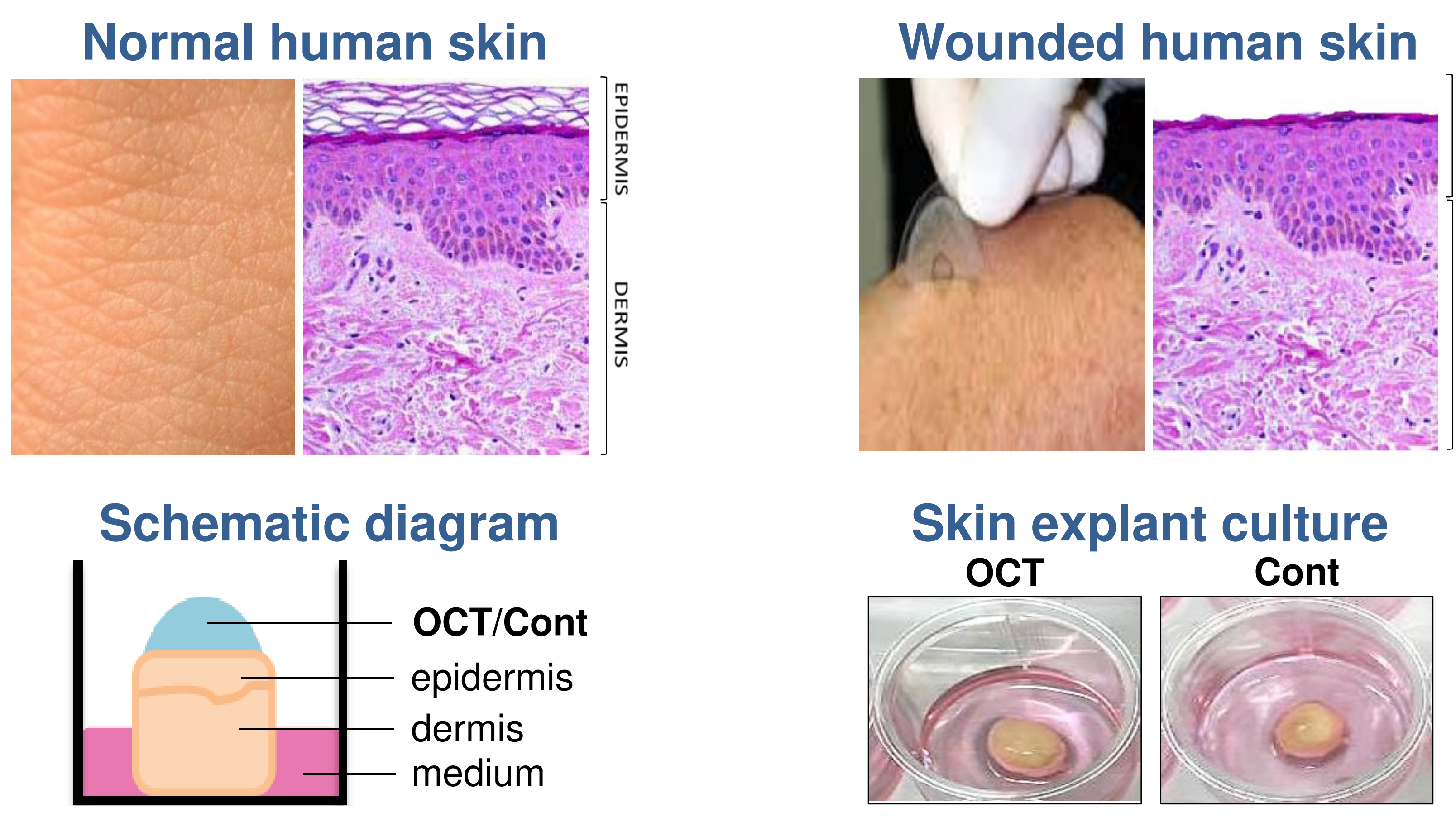

Fig 1. Depicted is normal and wounded skin from healthy human donors obtained during plastic surgery procedures, a schematic diagram and a photograph of the skin explant culture setup. OCT (=octenilin ${ }^{\circledR}$ ge hydrogel containing $0.05 \%$ octenidine; Schülke \& Mayr GmbH, GER) and control gel (=Normlge ${ }^{\circledR}$, Cont, hydrogel containing $0.9 \% \mathrm{w} / \mathrm{w}$ sodium chloride; Mölnlycke Health Care, SWE) were pipetted onto the epidermis of norma and wounded (tape-stripping, 50x) skin biopsies $(\varnothing=8 \mathrm{~mm})$ and cultured at defined time points $(24,48$ and $72 \mathrm{~h})$.

\section{Results}

OCT neither alters anatomy nor enhances apoptosis in skin cells upon wounding

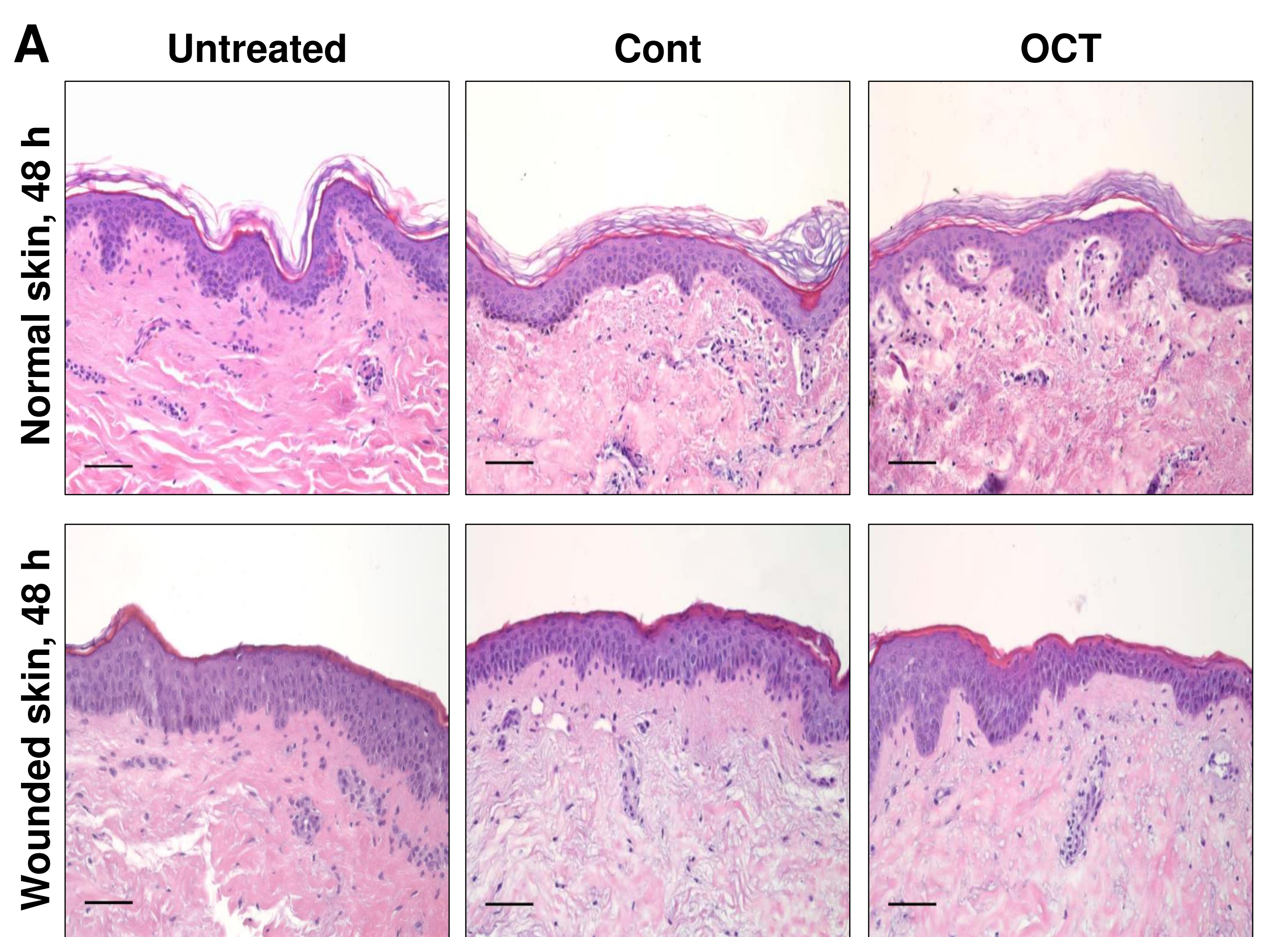

B Normal skin, before culture

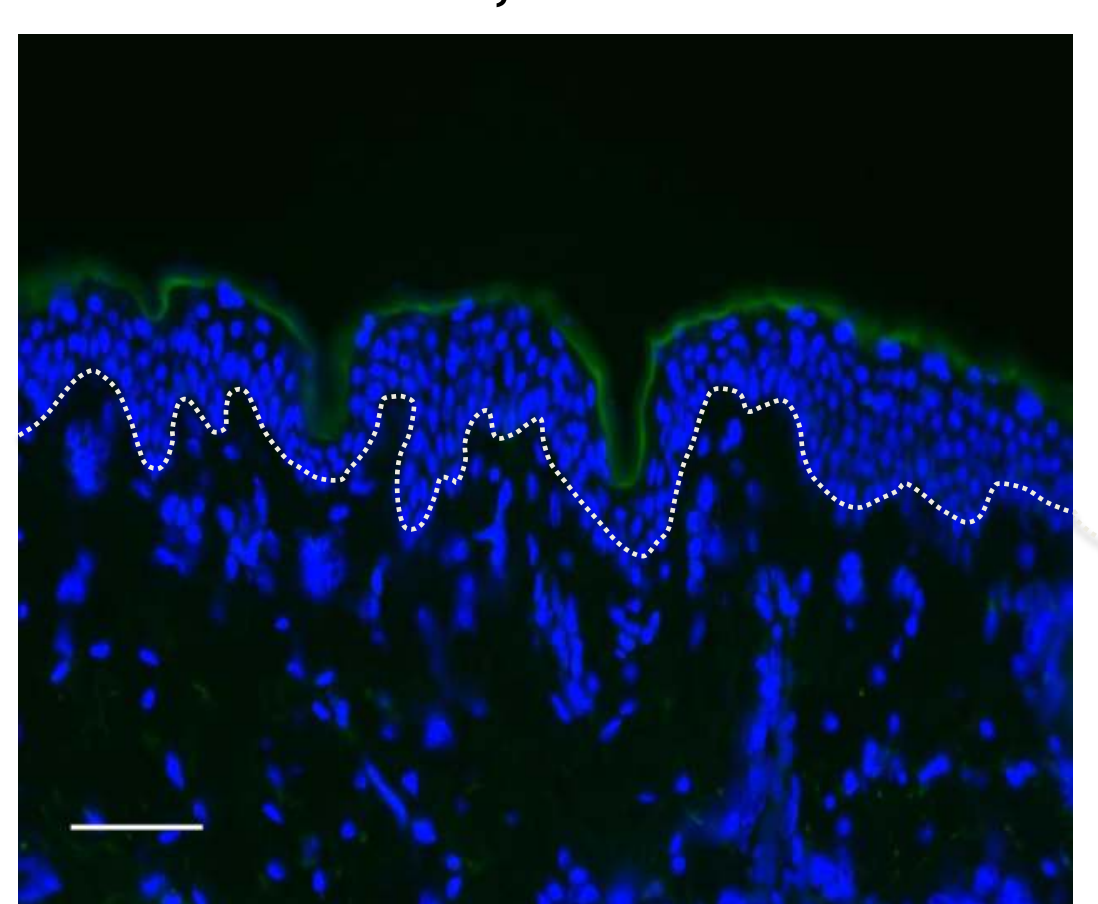

Wounded skin $48 \mathrm{~h}$, Cont
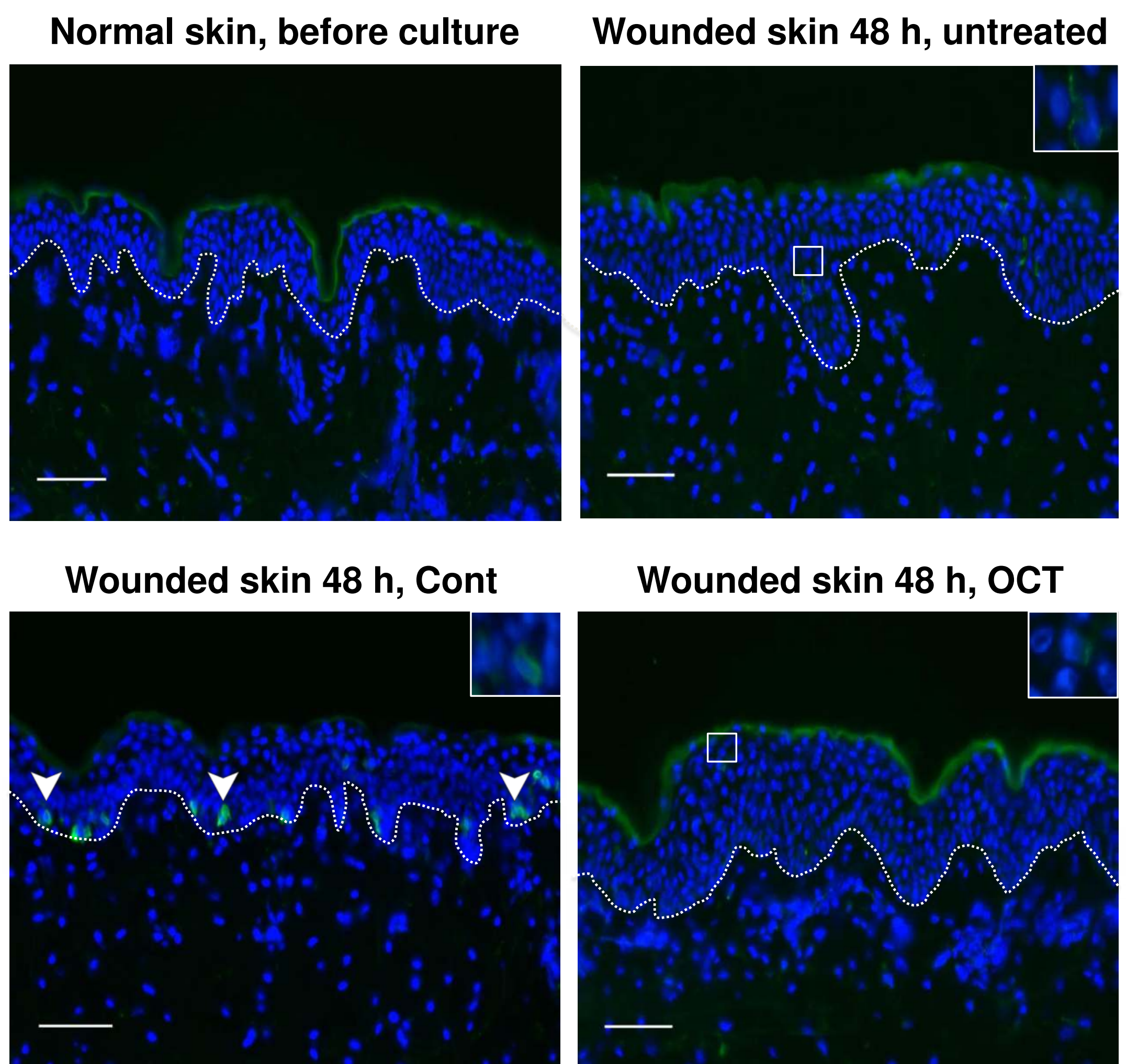

Wounded skin $48 \mathrm{~h}$, OCT

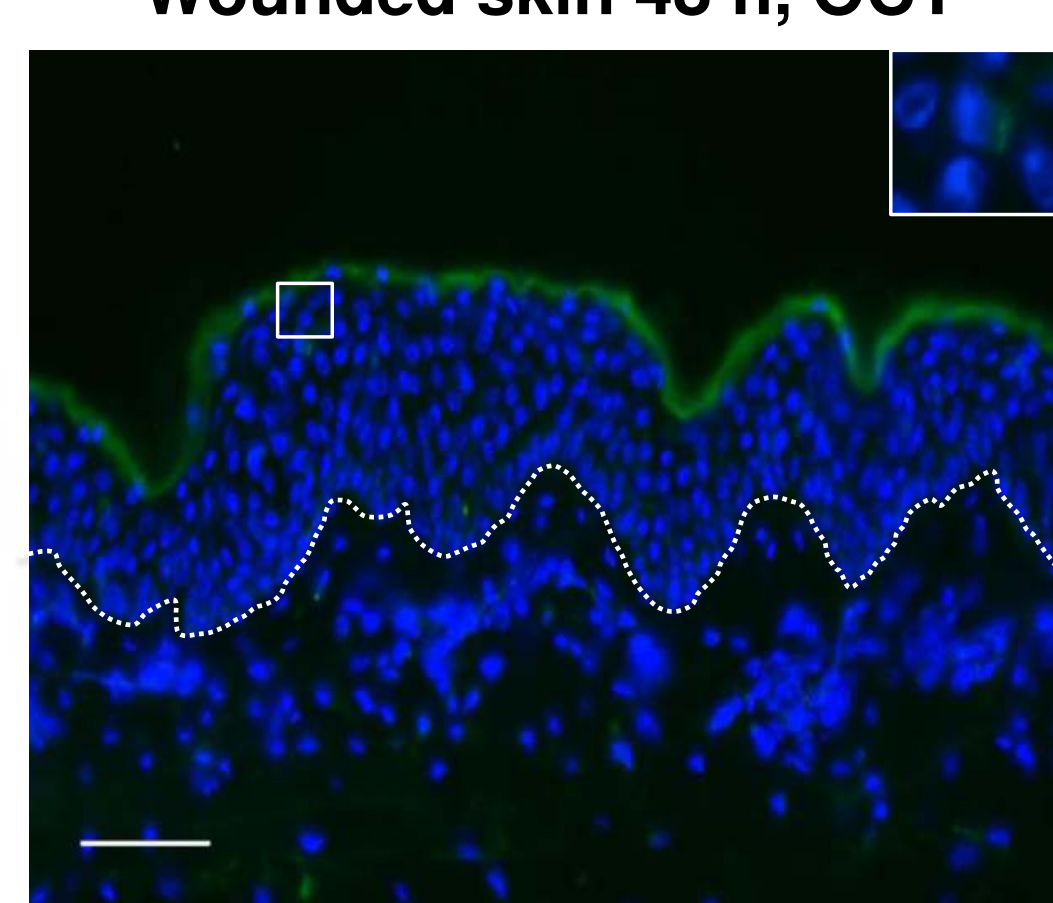

Fig 2. H\&E-stained paraffin sections (A), and active caspase 3 (green)-stained cryosections (DAPI, nuclear stain, blue) (B), of normal and wounded skin upon topical application of OCT/control gel and $48 \mathrm{~h}$ of culture. One representative donor is shown $(n=7)$. Scale bar $=200 \mu \mathrm{m}$.
OCT does not change LC morphology but prevents LC emigration and inhibits CD83 upregulation upon wounding
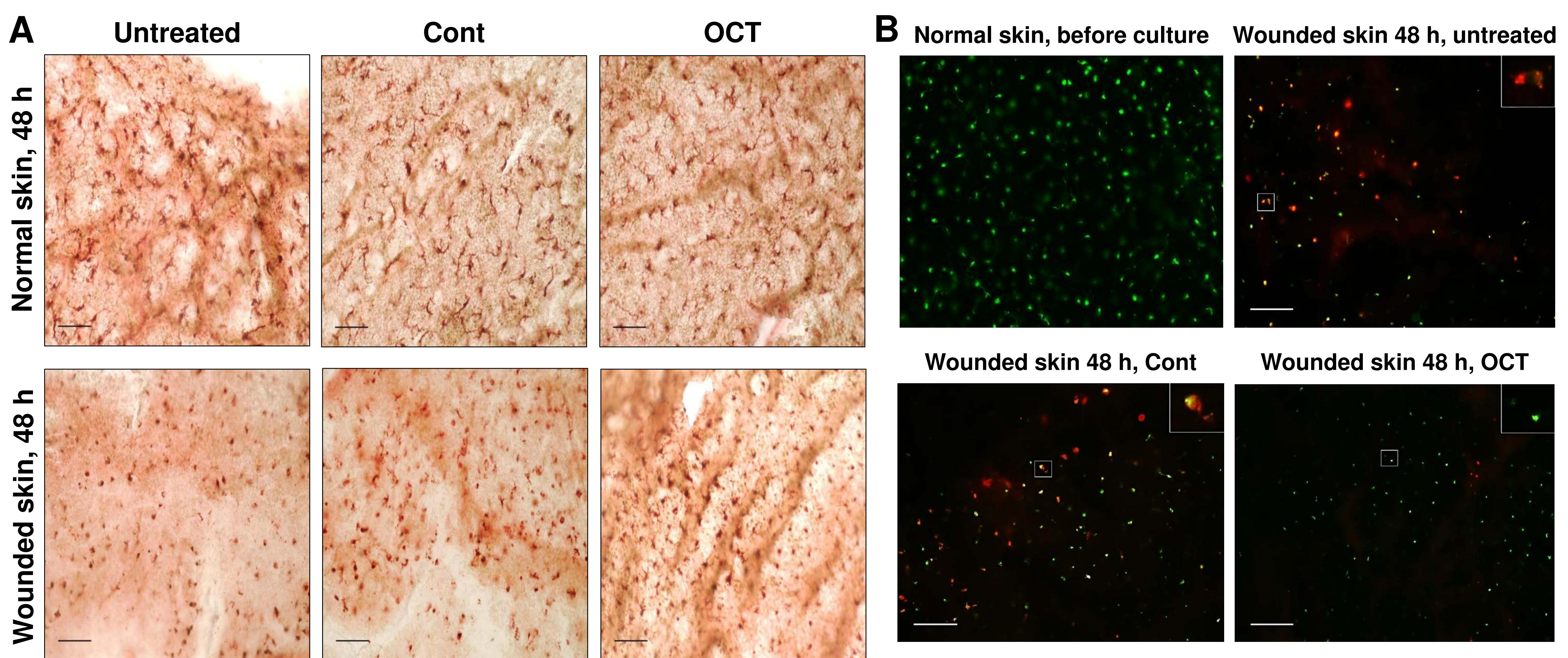

Wounded skin $48 \mathrm{~h}$, Cont

Wounded skin 48 h, OCT
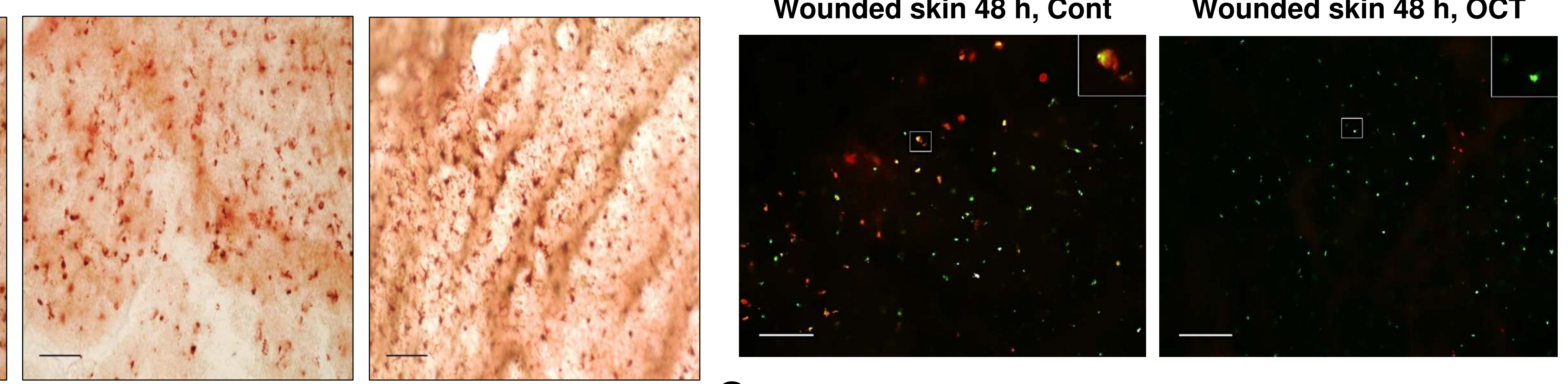

C

Fig 3. En face images of CD207+ (brown) LCs (A), and CD207+ (green) $\mathrm{CD}_{33^{+}}$(red) LCs (B), in epidermal sheets isolated from $48 \mathrm{~h}$ cultured normal and wounded skin with indicated treatments. One representative donor is shown $(n=7)$. Scale bar=200 $\mu$ m. Numbers of emigrated CD207+ LCs (C), and CD207+CD83+ LCs (D) in wounded skin upon $48 \mathrm{~h}$ of culture are indicated. Unpaired t-test, ${ }^{*} \mathrm{P} \leq 0.05$.

OCT significantly inhibits the secretion of IL-8 and IL-33 but not VEGF
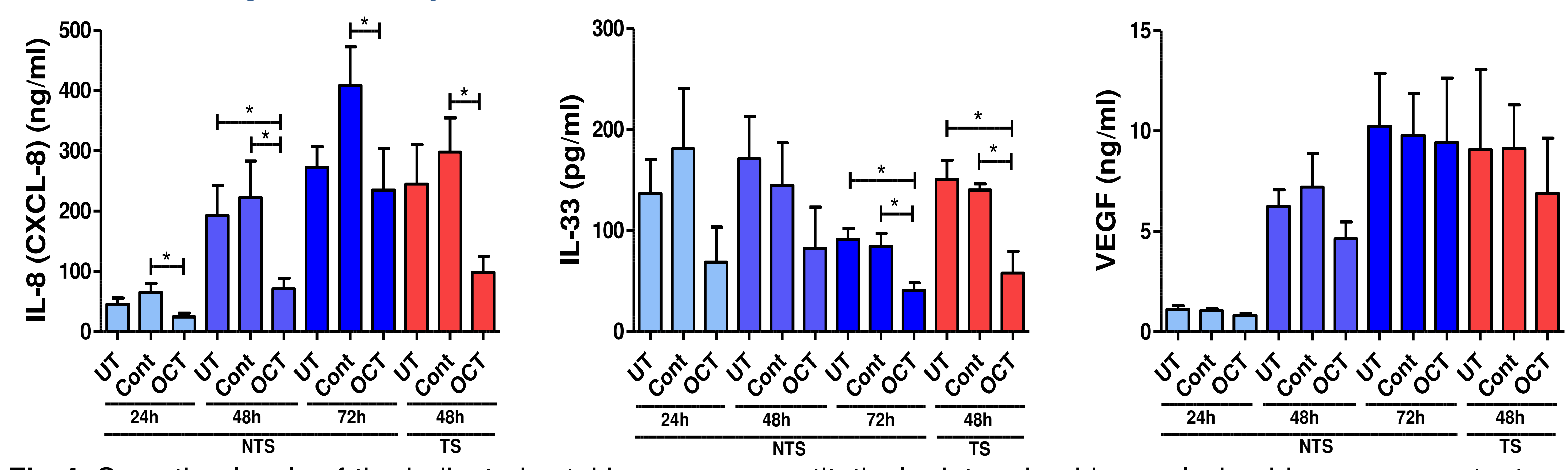

Fig 4. Secretion levels of the indicated cytokines were quantitatively determined by analyzing biopsy supernatants with ELISA. Data presented are mean $\pm S D(n=6)$ performed in triplicates. Unpaired $t$-test, ${ }^{*} P \leq 0.05$. NTS=normal skin, TS=wounded skin, UT=untreated skin, Cont=control gel, OCT=octenilin ${ }^{\circledR}$ gel.

\section{Conclusion}

Our data provide novel insights into the host response to OCT in the biologically relevant environment of viable human (wounded) skin, suggesting, in addition to its known antimicrobial activity, also an antiinflammatory action that might contribute to its observed positive wound healing influence resulting in better scar quality.

\section{Acknowledgements}

P. Hagenbach, K. Prpa, R. Reitermeier

C. Schuster, C. Klaus, K. Riedl 